\title{
Alterações Precoces nas Interleucinas Circulantes e no Risco Inflamatório Residual após Infarto Agudo do Miocárdio
}

\author{
Early Changes in Circulating Interleukins and Residual Inflammatory Risk After Acute Myocardial Infarction
}

\author{
Maria E. R. Coste, ${ }^{1}$ Carolina N. França, ${ }^{2}$ Maria Cristina Izar, ${ }^{1}{ }^{\circledR}$ Daniela Teixeira, ${ }^{1}$ Mayari E. Ishimura, ${ }^{1}$ leda Longo- \\ Maugeri, ${ }^{1}$ Amanda S. Bacchin, ${ }^{1}$ Henrique Tria Bianco, ${ }^{10}$ Flavio T. Moreira, ${ }^{1}$ Ibraim Masciarelli Pinto, ${ }^{3}$ Gilberto Szarf, ${ }^{1}$ \\ Adriano Mendes Caixeta, ${ }^{1}$ Otavio Berwanger, ${ }^{4}$ Iran Gonçalves Jr., ${ }^{1}$ Francisco A. H. Fonseca ${ }^{10}$ \\ Universidade Federal de São Paulo, ${ }^{1}$ São Paulo, SP - Brasil \\ Universidade de Santo Amaro, ${ }^{2}$ São Paulo, SP - Brasil \\ Instituto Dante Pazzanese de Cardiologia, ${ }^{3}$ São Paulo, SP - Brasil \\ Hospital Israelita Albert Einstein, ${ }^{4}$ São Paulo, SP - Brasil
}

\section{Resumo}

Fundamento: Pacientes com infarto agudo do miocárdio podem apresentar uma grande área infartada e disfunção ventricular mesmo com trombólise e revascularização precoces.

Objetivo: Investigar o comportamento das citocinas circulantes em pacientes com infarto agudo do miocárdio com supradesnivelamento do segmento ST (IAMCSST) e a relação delas com a função ventricular.

Métodos: No estudo BATTLE-AMI (Avaliação dos Linfócitos Tipos B e T no Infarto Agudo do Miocárdio), os pacientes com IAMCSST foram tratados com uma estratégia farmacoinvasiva. Os níveis de citocinas (IL-1 $\beta$, IL-4, IL-6, IL-10 e IL-18) no plasma foram testados através de ensaio de imunoadsorção enzimática (ELISA) no início do estudo e após 30 dias. A massa infartada e a fração de ejeção ventricular esquerda (FEVE) foram examinadas por ressonância magnética cardíaca 3-T. Valores de $\mathrm{p}$ menores que $\mathbf{0 , 0 5}$ foram considerados significativos.

Resultados: Na comparação com o início do estudo, níveis mais baixos foram detectados para IL-1 $\beta$ ( $p=0,028)$ e IL-18 $(p<0,0001)$ após 30 dias do IAMCSST, enquanto níveis mais altos foram observados para IL-4 ( $p=0,001)$ e IL-10 ( $p<0,0001)$ no mesmo momento. Em contrapartida, nenhuma mudança foi detectada nos níveis de IL-6 $(p=0,63)$. Os níveis da proteína C-reativa de alta sensibilidade e de IL-6 se correlacionaram no início do estudo $($ rho $=0,45, p<0,0001)$ e 30 dias após o IAMCSST ( $r h o=0,29, p=0,009)$. No início do estudo, a correlação entre os níveis de IL-6 e FEVE também foi observada (rho = -0,50, $p=0,004$ ).

Conclusões: Durante o primeiro mês pós-infarto agudo do miocárdio, observamos uma melhora significativa no balanço das citocinas pró e anti-inflamatórias, exceto da IL-6. Esses achados sugerem risco inflamatório residual. (Arq Bras Cardiol. 2020; 115(6):1104-1111)

Palavras-chave: Infarto do Miocárdio com Supradesnível do Segmento ST; Interleucina-6; Interleucina 10; Interleucina 18; Proteína C Reativa; Espectroscopia de Ressonância Magnética.

\section{Abstract}

Background: Patients with acute myocardial infarction may have a large infarcted area and ventricular dysfunction despite early thrombolysis and revascularization.

Objective: To investigate the behavior of circulating cytokines in patients with ST-segment elevation myocardial infarction (STEMI) and their relationship with ventricular function.

Methods: In the BATTLE-AMI (B and T Types of Lymphocytes Evaluation in Acute Myocardial Infarction) trial, patients with STEMI were treated with a pharmacoinvasive strategy. The plasma levels of cytokines (IL-1 $\beta, I L-4, I L-6, I L-10$, and IL-18) were tested using enzyme-linked immunosorbent assay (ELISA) at baseline and after 30 days. Infarcted mass and left ventricular ejection fraction (LVEF) were examined by 3-T cardiac magnetic resonance imaging. All p-values $<0.05$ were considered statistically significant.

Results: Compared to baseline, lower levels were detected for IL-1 $\beta(p=0.028)$ and IL-18 $(p<0.0001) 30$ days after STEMI, whereas higher levels were observed for IL-4 ( $p=0.001)$ and IL-10 ( $<$ 0.0001) at that time point. Conversely, no changes were detected for IL-6 levels $(p=0.63)$. The levels of high-sensitivity C-reactive protein and IL-6 correlated at baseline $(r h o=0.45, p<0.0001)$ and 30 days after STEMI (rho $=0.29, p=0.009)$. At baseline, correlation between IL-6 levels and LVEF was also observed (rho $=-0.50, p=0.004)$. Conclusions: During the first month post-MI, we observed a marked improvement in the balance of pro- and anti-inflammatory cytokines, except for IL-6. These findings suggest residual inflammatory risk. (Arq Bras Cardiol. 2020; 115(6):1104-1111)

Keywords: ST Elevation Myocardial Infarction; Interleukin-6, Interleukin-10, Interleukin-18; C-reactive Protein; Magnetic Resonance Spectroscopy. Full texts in English - http://www.arquivosonline.com.br

Correspondência: Francisco A. H. Fonseca •

Setor de Lípides, Aterosclerose e Blologia Vascular - Disciplina de Cardiologia - Rua Loefgren, 1350. CEP 04040-001, Vila Clementino, São Paulo, SP - Brasil E-mail: fahfonseca@terra.com.br

Artigo recebido em 22/08/2019, revisado em 01/12/2019, aceito em 27/12/2019

DOI: https://doi.org/10.36660/abc.20190567 


\section{Introdução}

Após o infarto agudo do miocárdio (IAM), os pacientes apresentam maiores taxas de hospitalização e morte devido a insuficiência cardíaca associada a maiores níveis de proteína C-reativa de alta sensibilidade (PCRas), mas esses eventos cardiovasculares podem ser reduzidos pela terapia anti-inflamatória. ${ }^{1}$ Foi descrito que a interleucina-6 (IL-6) tem um papel relevante na remodelação ventricular em modelos de sobrecarga de pressão ${ }^{2}$ e na coordenação da resposta imune após o IAM. ${ }^{3}$

Além da IL-6, outras interleucinas como a IL-18 e a IL-1 ${ }^{5,6}$ parecem contribuir para a remodelação ventricular adversa após o IAM. Curiosamente, a IL-4 parece contribuir para fibrose e disfunção ventricular na hipertensão arterial quando induzidas pela administração de angiotensina II. ${ }^{7}$ Em contrapartida, a IL-10 atenuou acentuadamente o microambiente inflamatório após o IAM, melhorando, assim, a função ventricular. ${ }^{8}$

Dado o interesse crescente no papel da inflamação na remodelação ventricular pós-IAM, examinamos a resposta inflamatória mediada por citocinas durante a fase precoce do IAM e a sua relação com a remodelação ventricular através de ressonância magnética cardíaca (RMC).

\section{Materiais e Métodos}

\section{População do estudo}

Este relato faz parte do estudo BATTLE-AMI (Avaliação dos Linfócitos Tipos B e T no Infarto Agudo do Miocárdio, ClinicalTrials.gov, NCT02428374). O BATTLE-AMI é um ensaio clínico randomizado no qual são comparados os efeitos da combinação de estatina e terapias antiplaquetárias na massa infartada e na fração de ejeção ventricular (FEVE) em pacientes com infarto agudo do miocárdio com supradesnivelamento do segmento ST (IAMCSST) tratados com uma estratégia farmacoinvasiva. ${ }^{9}$ Este estudo está em andamento e inclui pacientes com a primeira ocorrência de IAMCSST que foram submetidos a trombólise com tenecteplase nas primeiras 6 horas após o início dos sintomas e que foram transferidos para um hospital terciário (Hospital São Paulo) nas primeiras 24 horas para angiografia coronariana e procedimentos invasivos. Pacientes que tiveram eventos coronários prévios, revascularização da artéria coronária, contraindicações para RMC ou instabilidade hemodinâmica foram excluídos do estudo.

Este projeto foi aprovado pelo Comitê de Ética local (Universidade Federal de São Paulo, Hospital São Paulo, IRB:0297/2014, CAAE: 38692514.1.1001.5505), e todos os pacientes forneceram consentimento por escrito antes da inclusão.

\section{Exames laboratoriais}

Amostras de sangue foram coletadas na manhã do primeiro dia e entre 27-33 dias após o IAMCSST. Todas as amostras foram testadas no Laboratório de Lípides, Aterosclerose e Biologia Vascular (Universidade Federal de São Paulo). Os níveis plasmáticos de citocinas foram testados através do ensaio imunoadsorção enzimática (ELISA). IL-4, IL-6 e IL-10 foram testadas com os kits da BD Pharmingen (BD Biosciences, San Diego, Califórnia, EUA), e IL-1 $\beta$ e IL-18 foram testadas com os kits da R\&D (Minneapolis, Minnesota, EUA). Os resultados foram expressos em relação à absorbância usando o EnSpire Multimode Plate Reader (PerkinElmer) e/ou o iMark Microplate Absorbance Reader (Bio-Rad Laboratories, Hercules, Califórnia, EUA), de acordo com as instruções dos fabricantes. A proteína C-reativa de alta sensibilidade (PCRas) foi medida através de imunonefelometria.

\section{Ressonância Magnética Cardíaca}

Todas as imagens de RMC foram realizadas no Hospital São Paulo ou no Instituto Dante Pazzanese de Cardiologia. A primeira avaliação foi feita nos primeiros 10 dias (início do estudo), normalmente após a alta hospitalar. A segunda avaliação foi realizada de 27 a 33 dias após o IAM.

A quantidade de massa infartada, FEVE e microcirculação foram determinadas pela RMC 3-T. Para a função ventricular esquerda, as imagens de RMC foram adquiridas através de um scanner 3-T, como descrito anteriormente. ${ }^{9}$ Em resumo, a avaliação quantitativa foi realizada em uma estação de trabalho off-line, com o software Argus LV function (Siemens Healthineers). Para a quantificação da necrose miocárdica, a planimetria foi realizada com contorno manual das áreas com realce tardio de gadolínio, e o volume do tecido infartado foi calculado como a soma dessas áreas multiplicada pela espessura de cada corte.

A cine-RMC foi realizada com uma técnica de precessão livre no estado estacionário (imagens rápidas com uso de aquisição no estado estacionário). A isquemia foi detectada através de imagens de perfusão na primeira passagem apenas na orientação do eixo curto, com pelo menos três cortes (o número máximo de cortes é limitado pela frequência cardíaca). A detecção de infarto e a quantificação das imagens foram adquiridas através da técnica de realce tardio do miocárdio após a injeção de um agente de contraste à base de gadolínio (disponível comercialmente). As imagens com meio de contraste foram adquiridas nos mesmos planos que os usados para a cine-RMC, através de uma sequência segmentada com inversão da recuperação. Cada imagem de RMC foi revisada por dois especialistas cegados e independentes usando um software apropriado. A função do ventrículo esquerdo foi estimada com o uso de imagens de cine-RMC para medir os volumes de FEVE e de massa de acordo com métodos padrão. As imagens com realce tardio foram utilizadas para a caracterização do infarto. Em cada paciente, após o observador ter manualmente definido a região de interesse (RDI) em um território remoto e não infartado, o tecido miocárdico foi classificado como hiper-realçado (tecido fibroso) ou miocárdio realçado normalmente.

\section{Análise estatística}

Os dados são apresentados como média \pm desvio padrão ou mediana e interquartis (IQ), de acordo com a normalidade dos dados. Variáveis contínuas foram analisadas sob o aspecto da normalidade através do teste de Kolmogorov-Smirnov. 
As amostras basais e de 30 dias foram comparados com o teste não paramétrico de postos de Wilcoxon. As comparações entre os grupos foram feitas através do teste de Kruskal-Wallis. Os títulos de interleucinas e os parâmetros de RMC foram correlacionados através da análise de correlação de Spearman. O tamanho amostral foi estimado com base em estudos anteriores envolvendo alterações precoces nos títulos de interleucinas. ${ }^{10,11}$ O software SPSS, versão 18.0 (IBM, Armonk, Nova lorque, EUA), foi utilizado para a análise estatística. Valores de $p<0,05$ foram considerados estatisticamente significativos.

\section{Resultados}

\section{População do estudo}

Um total de 139 indivíduos consecutivos com IAMCSST foram incluídos no estudo. As principais características basais da população do estudo estão descritas na Tabela 1.

\section{Medição das citocinas circulantes}

A Figura 1 mostra que, em comparação com o início do estudo, os níveis de IL-1 $\beta$ e IL-18 diminuíram 30 dias após o IAMCSST. Em contrapartida, foram observados aumentos nos níveis de IL-4 e IL-10 em 30 dias após o IAMCSST. Não foram observadas mudanças significativas nos níveis de IL-6 nesse período.

Tabela 1 - Características basais da população do estudo

\begin{tabular}{|c|c|}
\hline Parâmetros & $N=139$ \\
\hline Idade, anos* & $56(50-63)$ \\
\hline Gênero masculino, n (\%) & $92(66)$ \\
\hline Tabagismo, n (\%) & $28(20)$ \\
\hline Diabetes, n (\%) & $32(24)$ \\
\hline $\mathrm{HbA} 1 \mathrm{c}, \%^{* *}$ & $6,4 \pm 1,4$ \\
\hline Hipertensão, n (\%) & $82(60)$ \\
\hline PAS $(\mathrm{mmHg})^{\star *}$ & $128 \pm 21$ \\
\hline $\operatorname{PAD}(\mathrm{mmHg})^{* *}$ & $80 \pm 14$ \\
\hline Peso, $\mathrm{kg}^{* *}$ & $75 \pm 13$ \\
\hline $\mathrm{IMC}, \mathrm{km} / \mathrm{m}^{2 * *}$ & $27,2 \pm 4,78$ \\
\hline Colesterol, mg/dL** & $208 \pm 45$ \\
\hline Colesterol LDL, mg/dL** & $138 \pm 41$ \\
\hline Colesterol HDL, mg/dL ${ }^{* *}$ & $41 \pm 12$ \\
\hline Triglicerídios, mg/dL* & $124(86-213)$ \\
\hline PCRas, mg/L* & $15(7-63)$ \\
\hline \multicolumn{2}{|l|}{ Localização do infarto } \\
\hline Anterior, n (\%) & $60(43)$ \\
\hline Inferior, n (\%) & $73(53)$ \\
\hline Lateral, n (\%) & $6(4)$ \\
\hline
\end{tabular}

*mediana (intervalo interquartil); **média \pm desvio padrão; HbA1c: hemoglobina glicada; HDL: lipoproteína de alta densidade; IMC: índice de massa corporal; LDL: lipoproteina de baixa densidade; PAD: pressão arterial diastólica PAS: pressão arterial sistólica; PCRas: proteína C-reativa de alta sensibilidade.

\section{Relação entre citocinas e ressonância magnética cardíaca}

No início do estudo, não foram observadas correlações significativas entre os níveis de IL-1 $\beta$, IL-4, IL-10 e IL-18 e os parâmetros de RMC, como a quantidade de massa infartada ou a FEVE; no entanto, houve uma correlação negativa entre os níveis de IL-6 e a FEVE (rho de Spearman $=-0,50$, $p=0,004)$. Uma tendência de correlação entre os níveis de IL-6 e a porcentagem de massa infartada do ventrículo esquerdo também foi observada ( $r$ o $=0,41, p=0,05$ ) (Tabela 2).

Houve uma correlação positiva entre os níveis basais de IL-4 e a quantidade de massa infartada medida pela RMC $(r h o=0,24 ; p=0,03$ ) em 30 dias (Tabela 3). Nenhuma outra correlação entre os níveis de citocinas e os parâmetros de RMC foi encontrada na avaliação de 30 dias após o IAMCSST.

\section{Relação entre citocinas e proteína C-reativa de alta sensibilidade}

Os níveis de PCRas se correlacionaram com os de IL-6 no início do estudo (rho $=0,45, p<0,0001)$ e 30 dias após o IAMCSST (rho $=0,29, p=0,009$ ). Nenhuma outra citocina mostrou correlação com os níveis de PCRas tanto no início do estudo quanto 30 dias após o IAMCSST (dados não apresentados).

\section{Relação entre IL-6 e artéria coronária culpada}

A artéria coronária direita foi a artéria coronária mais comumente culpada pelo IAMCSST (46\%), seguida pela artéria descendente anterior esquerda (42\%) e pela artéria circunflexa esquerda (12\%). Os níveis de IL-6 constatados no primeiro dia após o IAMCSST não foram diferentes entre as artérias culpadas ( $p=0,063$ no teste de Kruskal-Wallis), assim como 30 dias após o IAMCSST ( $p=0,131$ no teste de Kruskal-Wallis).

\section{Ressonância magnética cardíaca}

A Tabela 4 mostra os resultados da RMC de acordo com a artéria coronária culpada. Não foram observadas diferenças significativas para massa infartada (\%), massa ventricular esquerda ou FEVE no início do estudo ou 30 dias após o IAMCSST.

\section{Discussão}

Nosso estudo mostra o comportamento das concentrações de citocinas na fase precoce do IAMCSST em pacientes que foram tratados com uma estratégia farmacoinvasiva e receberam cuidado médico padrão. Nossos principais achados foram uma diminuição significativa nos títulos de citocinas pró-inflamatórias (IL-1ß e IL-18), mas não nos de IL-6, e um aumento nos títulos de citocinas protetoras (IL-10 e IL-4). Curiosamente, a FEVE obtida pela RMC mostrou correlação com as concentrações basais de IL-6, mas não 30 dias após o IAMCSST. Além disso, a massa infartada quantificada pela RMC no período de 30 dias mostrou correlação com os níveis basais de IL-4. Em conjunto, esses dados apontam para um papel importante do perfil das interleucinas no primeiro dia após o IAM, que parece apresentar relação com a massa infartada e a remodelação ventricular. Além disso, a diminuição substancial de algumas citocinas inflamatórias juntamente com o aumento significativo da IL-10 parece 
Artigo Original
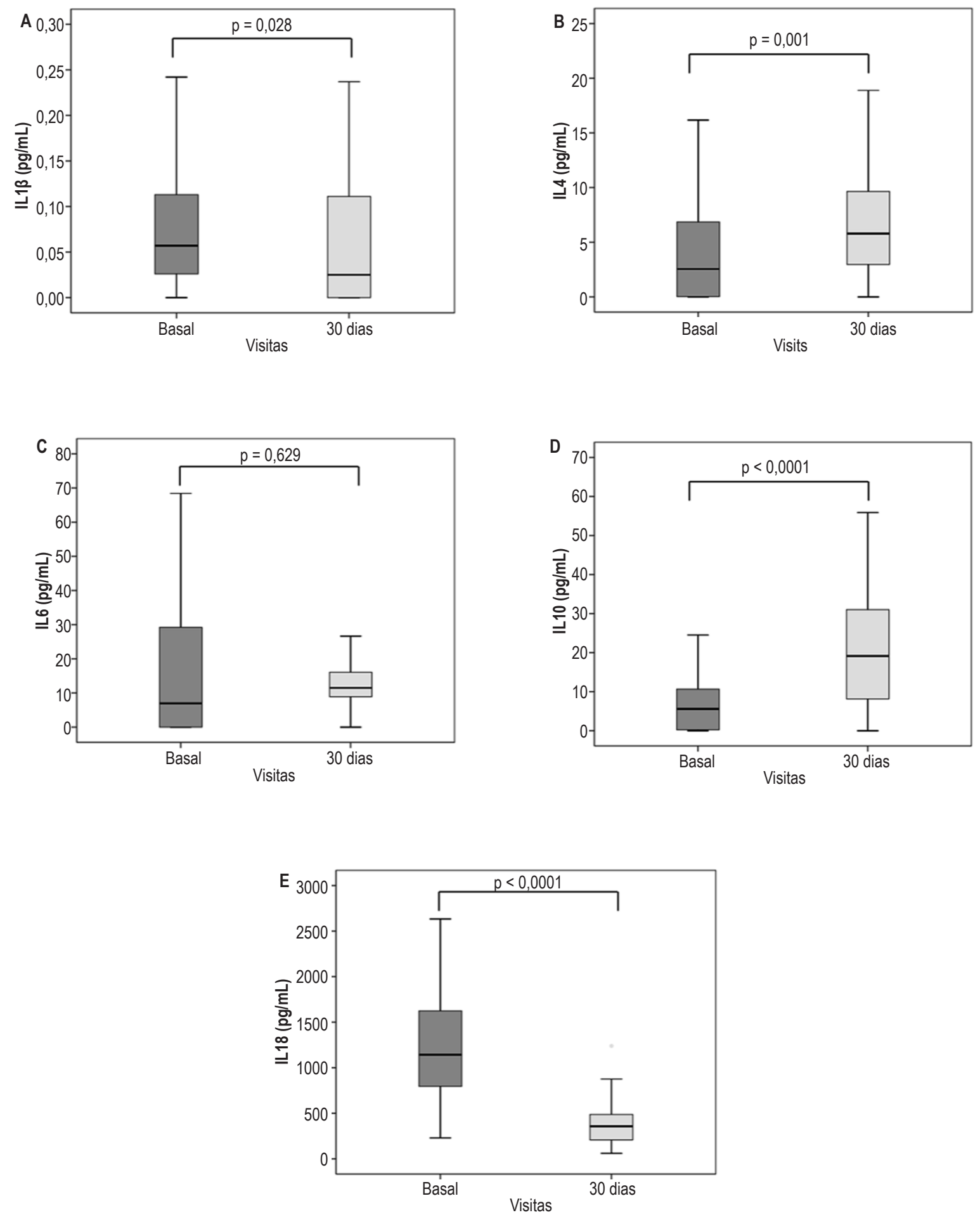

Figura 1 - Gráficos em caixa das concentrações de interleucina (IL) no basal e 30 dias após o IAMCSST. (A) IL-1B; (B) IL- 4; (C) IL-6; (D) IL-10; (E) IL-18. Alterações significativas foram observadas em todas as citocinas, exceto na IL-6. Títulos foram comparados pelo teste de Wilcoxon. 


\section{Artigo Original}

Tabela 2 - Correlações entre interleucinas basais $(\mathrm{pg} / \mathrm{mL}) \mathrm{e}$ parâmetros de ressonância magnética cardíaca na fase aguda do infarto do miocárdio

\begin{tabular}{|c|c|c|}
\hline Variáveis & rho de Spearman & Valor de $p$ \\
\hline IL-1 $1 \beta$ e massa infartada* & 0,16 & 0,43 \\
\hline IL-1 $\beta$ e massa infartada** & $-0,05$ & 0,84 \\
\hline IL-1 $1 \beta$ e FEVE & 0,12 & 0,55 \\
\hline IL-4 e massa infartada* & $-0,26$ & 0,19 \\
\hline IL-4 e massa infartada** & $-0,2$ & 0,37 \\
\hline IL-4 e FEVE & 0,15 & 0,44 \\
\hline IL-6 e massa infartada* & 0,16 & 0,39 \\
\hline IL-6 e massa infartada** & 0,41 & 0,05 \\
\hline IL-6 e FEVE & $-0,5$ & 0,004 \\
\hline IL-10 e massa infartada* & 0,3 & 0,1 \\
\hline IL-10 e massa infartada** & 0,24 & 0,28 \\
\hline IL-10 e FEVE & $-0,31$ & 0,09 \\
\hline IL-18 e massa infartada* & $-0,11$ & 0,57 \\
\hline IL-18 e massa infartada** & $-0,24$ & 0,28 \\
\hline IL-18 e FEVE & 0,01 & 0,96 \\
\hline
\end{tabular}

*gramas; ** porcentagem de massa ventricular esquerda; FEVE: fração de ejeção ventricular esquerda; IL: interleucina.
Tabela 3 - Correlações entre concentrações basais de interleucina $(\mathrm{pg} / \mathrm{mL})$ e parâmetros de ressonância magnética cardíaca 30 dias após o infarto do miocárdio

\begin{tabular}{lcc}
\hline Variáveis & rho de Spearman & Valor de $\mathbf{p}$ \\
\hline IL-1 $\beta$ e massa infartada* & $-0,02$ & 0,85 \\
IL-1 $\beta$ e massa infartada** & $-0,07$ & 0,59 \\
IL-1 $\beta$ e FEVE & 0,19 & 0,1 \\
IL-4 e massa infartada* & 0,24 & 0,03 \\
IL-4 e massa infartada** & 0,14 & 0,2 \\
IL-4 e FEVE & $-0,14$ & 0,19 \\
IL-6 e massa infartada* & 0,13 & 0,23 \\
IL-6 e massa infartada ${ }^{* *}$ & 0,13 & 0,22 \\
IL-6 e FEVE & $-0,17$ & 0,1 \\
IL-10 e massa infartada* & 0,13 & 0,23 \\
IL-10 e massa infartada** & 0,07 & 0,55 \\
IL-10 e FEVE & 0,09 & 0,4 \\
IL-18 e massa infartada* & 0,14 & 0,31 \\
IL-18 e massa infartada** & $-0,12$ & 0,38 \\
IL-18 e FEVE & 0,07 & 0,52 \\
\hline
\end{tabular}

"gramas; ** porcentagem de massa ventricular esquerda; FEVE: fração de ejeção ventricular esquerda; IL: interleucina.

Tabela 4 - Resultados da ressonância magnética cardíaca por artéria coronária culpada no início do estudo e 30 dias após infarto agudo do miocárdio

\begin{tabular}{|c|c|c|}
\hline Artéria coronária culpada & Início & 30 dias \\
\hline \multicolumn{3}{|c|}{ Artéria descendente anterior esquerda } \\
\hline Tamanho do infarto, \% VE & $10,0(5,5-19,0)$ & $12,7(8,0-21,0)$ \\
\hline Massa do VE, gramas & $117,3(101,0-171,8)$ & $90,5(69,0-127,9)$ \\
\hline FEVE, \% & $46,0(43,3-59,0)$ & $51,6(40,5-59,3)$ \\
\hline \multicolumn{3}{|l|}{ Artéria coronária direita } \\
\hline Tamanho do infarto, \% VE & $12,0(10,0-18,5)$ & $10,0(6,0-17,9)$ \\
\hline Massa do VE, gramas & $96,0(86,5-123,0)$ & $99,0(80,0-113,0)$ \\
\hline FEVE, \% & $48,0(42,0-50,5)$ & $55,5(50,0-60,0)$ \\
\hline \multicolumn{3}{|l|}{ Artéria circunflexa esquerda } \\
\hline Tamanho do infarto, \% VE & $11,5(5,0-18,0)$ & $7,0(4,0-8,7)$ \\
\hline Massa do VE, gramas & $103,0(76,0-130,0)$ & $103,0(75,0-106)$ \\
\hline FEVE, \% & $51,0(50,0-52,0)$ & $54,0(51,0-58,0)$ \\
\hline
\end{tabular}

Dados apresentados como mediana (intervalo interquartil). FEVE: fração de ejeção ventricular esquerda; VE: ventrículo esquerdo. As imagens basais de ressonância magnética cardíaca foram obtidas em até 10 dias após o infarto do miocárdio. No início do estudo, nenhuma diferença foi observada no tamanho do infarto entre as artérias coronárias culpadas $(p=0,59)$, assim como na massa do VE $(p=0,08)$ ou na FEVE $(p=0,62)$ (teste de Kruskal-Wallis para todas as análises). Também não foram observadas diferenças entre as artérias coronárias culpadas depois de 30 dias no tamanho do infarto $(p=0,13)$, na massa do VE $(p=0,86)$ ou na FEVE $(p=0,10)$ (o teste de Kruskal-Wallis foi usado nessas comparações). 
atenuar, ao menos em parte, os efeitos prejudiciais da IL-6 na remodelação ventricular. ${ }^{12}$

A recanalização coronária precoce e o uso tanto de fármacos antitrombóticos quanto de hipolipemiantes altamente efetivos são estratégias bem estabelecidas no tratamento de pacientes com IAM. No entanto, um melhor conhecimento do risco inflamatório residual durante o acompanhamento inicial de IAM pode contribuir para novas oportunidades terapêuticas. ${ }^{13}$

No nosso estudo, as concentrações de IL-6 foram inversamente associadas à função ventricular esquerda. Estudos de randomização mendeliana sugeriram um papel causal da IL-6 na coronariopatia ${ }^{14,15}$ e no desenvolvimento de aneurisma de aorta abdominal. ${ }^{16}$ Dois grandes estudos prospectivos recentes envolvendo indivíduos após eventos coronários agudos mostraram uma associação independente entre maiores concentrações de IL-6 e desfechos cardiovasculares principais, incluindo morte cardiovascular, mesmo após múltiplos ajustes para os biomarcadores clássicos de doença cardiovascular. ${ }^{17,18}$ A interação da IL-6 com seu receptor parece modular o microambiente inflamatório em doenças cardiovasculares tanto em relação à desestabilização da placa quanto ao prognóstico de longo prazo.

Esse microambiente inflamatório, que envolve os biomarcadores endoteliais e inflamatórios, pode ser modulado pela terapia antiplaquetária escolhida. ${ }^{19,20}$ No entanto, no estudo DISPERSE-2 (Estudo de Confirmação de dose para Avaliação dos Efeitos Antiplaquetários de AZD6140 vs. Clopidogrel no IAMSSST 2), ${ }^{21}$ ao comparar o uso de ticagrelor com clopidogrel após síndrome coronariana aguda recente, não foram encontradas diferenças nos biomarcadores inflamatórios no início do estudo, na alta hospitalar e após 4 semanas. É possível que a diminuição acentuada nos níveis de outros marcadores inflamatórios, como IL-1 $\beta$ e IL-18, e o aumento nos níveis de IL-10 protetora tenham contribuído para uma resposta inflamatória mais favorável, apesar dos níveis persistentemente elevados de IL-6. Além disso, esses pacientes receberam tratamento hipolipemiante efetivo de ação anti-inflamatória comprovada, com rosuvastatina ${ }^{22}$ ou com uma combinação de sinvastatina e ezetimiba. ${ }^{23}$ Curiosamente, apenas o nível de IL-6 não se alterou após o tratamento médico, o que sugere que o controle dessa citocina requer terapia adicional, como o uso de anticorpo monoclonal ou de um fármaco que reduza a atividade inflamatória desencadeada pela IL-6. ${ }^{24,25}$

A IL-4 tem diversas propriedades biológicas, incluindo a diferenciação dos linfócitos Th1 em células com atividade inflamatória menor (Th2). ${ }^{25}$ Além disso, foi relatado que a IL-4 cronicamente elevada tem uma relação causal com a fibrose cardíaca e a remodelação cardíaca adversa. ${ }^{26}$ Além disso, a cardiomiopatia dilatada induzida pela angiotensina II é modulada pelos níveis de IL-4. ${ }^{27}$ Neste estudo, observamos uma associação entre os níveis basais de IL-4 e a quantidade de massa infartada depois de 30 dias do IAM. Esses achados sugerem um papel importante dessa citocina, que possivelmente atenua o processo inflamatório miocárdico através de maior diferenciação celular em fenótipos menos inflamatórios (macrófagos M2 e linfócitos Th2). Nesse cenário, a IL-4 pode influenciar todo o processo de remodelação ventricular. Este pode levar diversas semanas para ocorrer e parece depender da relação entre células inflamatórias e cardiomiócitos, determinando, assim, a eliminação de células necróticas e promovendo reposição celular e formação de tecido fibroso. ${ }^{28}$

Os inflamassomas são uma família do sistema imune inato que inclui o NLRP3, que foi reconhecido como um gatilho relevante para o efeito inflamatório em cascata relacionado à doença cardiovascular. ${ }^{29}$ Essa plataforma pode ser ativada por diversos estímulos, incluindo a hipóxia, promovendo a liberação das citocinas altamente inflamatórias IL-1 $\beta$ e IL-18. ${ }^{30}$ Além disso, a síndrome metabólica e o diabetes estão relacionados às concentrações de IL-18. Enquanto a IL-1 $\beta$ está relacionada ao efeito inflamatório em cascata da doença cardiovascular, a IL-18 parece estar associada a mecanismos inflamatórios, favorecendo o desenvolvimento de câncer e apresentando maiores concentrações em pacientes com diabetes e resistência à insulina. ${ }^{31,32}$ Nosso estudo mostrou uma diminuição nas duas citocinas (IL-1 $\beta$ e IL-18), o que sugere uma diminuição nos estímulos para ativação do NLRP3 após 30 dias do IAMCSST.

Este estudo também reforça a importância do papel da IL-6, a única citocina não modificada após 30 dias do IAM, que apresentou uma correlação significativa com a PCRas basal e 30 dias após o IAMCSST, uma associação previamente relatada. ${ }^{33}$ No nosso estudo, apenas pacientes com IAMCSST submetidos a trombólise nas primeiras 6 horas e encaminhados para angiografia coronariana nas primeiras 24 horas foram incluídos. Assim, esta é uma população altamente homogênea que recebeu cuidado médico padrão. Levando em consideração a associação de níveis cronicamente elevados de IL-6 e a recorrência de eventos coronários, insuficiência cardíaca, mortalidade cardiovascular e mortalidade por todas as causas, uma diminuição adicional no risco inflamatório residual parece ser um alvo promissor para a intervenção. ${ }^{34,35}$

\section{Limitações do estudo}

A população estudada recebeu terapia hipolipemiante e antiplaquetária, cujos efeitos anti-inflamatórios podem ter contribuído para os resultados do estudo. No entanto, esses tratamentos são parte do cuidado padrão desses pacientes. Algumas citocinas inflamatórias capazes de ativar a via inflamatória mediada pela IL-6, como o fator de necrose tumoral-alfa (TNF- $\alpha$ ) ou o IL-1R, não foram medidas e podem ter relevância nas respostas teciduais e na remodelação ventricular do paciente. ${ }^{36,37} \mathrm{Na}$ verdade, o IAM per se pode estar relacionado a um aumento na IL-6 como resposta a uma lesão. No entanto, os títulos de IL-6 permaneceram elevados enquanto outras citocinas alteraram seus níveis séricos após 30 dias do IAMCSST. Outro biomarcador inflamatório importante que não foi avaliado neste estudo é a IL-1 $\alpha$, que é liberada pelos cardiomiócitos necróticos e ativa as respostas imunes dos fibroblastos. ${ }^{38} \mathrm{O}$ bloqueio da IL-1a diminui a atividade quimiotática para diversas células mediadas por CCL2/ MCP-1 e IL-6. ${ }^{38}$ Além disso, o recrutamento de monócitos e linfócitos na isquemia cardíaca pode ser estimulado por diversos quimiotáticos, como CCL2 e CCL5, influenciando, assim, a cura tecidual. ${ }^{39}$ Por fim, o fator de transformação do 
crescimento beta (TGF- $\beta$ ), que é altamente expressado após o IAM, também não foi avaliado pelo nosso estudo, mas foi implicado na sobrevida de cardiomiócitos e na remodelação ventricular. ${ }^{40}$ Os resultados apresentados aqui referem-se a um período relativamente precoce após o IAM, mas é quando os infiltrados inflamatórios parecem mais relevantes para a recuperação celular ou lesão de reperfusão.

\section{Conclusões}

Durante o primeiro mês pós-IAM, observamos uma melhora significativa no balanço das citocinas pró e antiinflamatórias, exceto da IL-6. Esses achados sugerem risco inflamatório residual.

\section{Destaques}

- As estratégias atuais de cuidado de pacientes com infarto agudo do miocárdio parecem insuficientes para modificar a via inflamatória mediada pela interleucina-6.

- Maiores concentrações dessa citocina parecem estar associadas a menor fração de ejeção ventricular.

- As terapias direcionadas à interleucina-6 parecem promissoras para diminuição adicional do risco inflamatório residual em sujeitos com infarto agudo do miocárdio.

- O grande desafio de reduzir o risco inflamatório residual está em desenvolver terapias seguras e acessíveis.

\section{Contribuição dos autores}

Concepção e desenho da pesquisa: Izar MC, Maugeri IL, Pinto IM, Szarf G, Caixeta AM, Berwanger O, Fonseca FAH; Obtenção de dados: Coste MER, Izar MC, Teixeira D, Ishimura ME, Bacchin AS, Bianco HT, Moreira FT, Pinto IM, Szarf G, Caixeta AM, Gonçalves Jr. I, Fonseca FAH; Análise e interpretação dos dados: Coste MER, França CN, Izar MC, Teixeira D, Ishimura ME, Maugeri IL, Bacchin AS, Bianco HT, Moreira FT, Pinto IM, Szarf G, Caixeta AM, Berwanger $\mathrm{O}$, Gonçalves Jr. I, Fonseca FAH; Análise estatística: Coste MER, França CN, Fonseca FAH; Obtenção de financiamento: Fonseca FAH; Redação do manuscrito: Coste MER, Fonseca FAH; Revisão crítica do manuscrito quanto ao conteúdo intelectual importante: Izar MC, Maugeri IL, Fonseca FAH.

\section{Potencial conflito de interesses}

Declaro não haver conflito de interesses pertinentes.

\section{Fontes de financiamento}

O presente estudo foi financiado pela FAPESP (2012/516927) e CNPq 300937/2015-6 e 428793/2016-9. Drogas antiplaquetárias e hipolipemiantes foram obtidas através de um estudo de iniciativa do investigador pela AstraZeneca (ESR 14-10726), que não influenciou a concepção do estudo, a coleta de dados, a análise estatística e as publicações.

\section{Vinculação acadêmica}

Este artigo é parte de dissertação de Mestrado de Maria E. R. Coste pela Universidade Federal de São Paulo.

\section{Referências}

1. Everett BM, Cornel J, Lainscak M, Anker SD, Abbate A, Thuren T, et al. Anti-inflammatory therapy with Canakinumab for the prevention of hospitalization for heart failure. Circulation. 2019;139(10):1289-99.

2. Zhao L, Cheng G, Jin R, Afzal MR, Samanta A, Xuan YT, et al. Deletion of interleukin-6 attenuates pressure overload-induced left ventricular hypertrophy and dysfunction. Circ Res. 2016;118(12):1918-29.

3. Huang M, Yang D, Xiang M, Wang J. Role of interleukin-6 in regulation of immune responses to remodeling after myocardial infarction. Heart Fail Rev. $2015 ; 20(1): 25-38$

4. Su Z, Lin R, Chen Y, Shu X, Zhang H, Nie R, et al. Knockdown of EMMPRIN improves adverse remodeling mediated by IL-18 in the post-infarcted heart. Am J Transl Res. 2015;7(10):1908-16.

5. Suetomi T, Willeford A, Brand CS, Cho Y, Ross RS, Miyamoto S, et al Inflammation and nlrp3 inflammasome activation initiated in response to pressure overload by $\mathrm{ca}_{2+} / \mathrm{calmodulin}$-dependent protein kinase ii $\delta$ signaling in cardiomyocytes are essential for adverse cardiac remodeling. Circulation. 2018;138(22):2530-44

6. Ørn S, Ueland T, Manhenke C, Sandanger Ø, Godang K, Yndestad A et al. Increased interleukin-1 $\beta$ levels are associated with left ventricula hypertrophy and remodelling following acute ST segment elevation myocardial infarction treated by primary percutaneous coronary intervention. J Intern Med. 2012;272(3):267-76.

7. Peng H, Sarwar Z, Yang XP, Peterson EL, Xu J, Janic B, et al. Profibrotic role for interleukin-4 in cardiac remodeling and dysfunction. Hypertension. 2015;66(3):582-9.

8. Jung M, Ma Y, lyer RP, DeLeon-Pennell KY, Yabluchiansky A, GarrettMR, et al. IL-10 improves cardiac remodeling after myocardial infarction by stimulating M2 macrophage polarization and fibroblast activation. Basic Res Cardiol. 2017;112(3):33.

9. Fonseca FAH, Izar MC, Maugeri IML, Berwanger O, Damiani LP, Pinto IM, et al. Effects of four antiplatelet/statin combined strategies on immune and inflammatory responses in patients with acute myocardial infarction undergoing pharmacoinvasive strategy: design and rationale of the B and $T$ Types of lymphocytes evaluation in acute myocardial infarction (BATTLEAMI) study: study protocol for a randomized controlled trial. Trials. 2017 Dec 19;18:601.

10. Gong X, Zhou R, Li Q. Effects of captopril and valsartan on ventricular remodeling and inflammatory cytokines after interventional therapy for AMI. Exp Ther Med. 2018;16(4):3579-83.

11. Morariu M, Hodas R, Benedek T, Benedek I, Opincariu D, Mester A, et al. Impact of inflammation-mediated response on pan-coronary plaque vulnerability, myocardial viability and ventricular remodeling in the postinfarction period - the VIABILITY study: protocol for a non-randomized prospective clinical study. Medicine (Baltimore). 2019;98(17):e15194.

12. Kotter S, Kazmierowska M, Andresen C, Bottermann K, Grandoch M, Gorressen S, et al. Titin-based cardiac myocyte stiffening contributes to early adaptive ventricular remodeling after myocardial infarction. Circ Res. 2016;119(9):1017-29.

13. Ridker PM. From CRP to IL-6 to IL-1: moving upstream to identify novel targets for atheroprotection. Circ Res. 2016;118(1):145-56 
14. Interleukin-6 Receptor Mendelian Randomisation Analysis (IL6R MR) Consortium, Swerdlow DI, Holmes MV, Kuchenbaecker KB, Engmann JEL, Shah T, et al. The interleukin- 6 receptor as a target for prevention of coronary heart disease: a mendelian randomisation analysis. Lancet. 2012;379(9822):1214-24.

15. IL6R Genetics Consortium Emerging Risk Factors Collaboration, Sarwar N, Butterworth AS, Freitag DF, Gregson J, Willeit P, et al. Interleukin-6 receptor pathways in coronary heart disease: a collaborative meta-analysis of 82 studies. Lancet. 2012;379(9822):1205-13.

16. Harrison SC, Smith AJ, Jones GT, Swerdlow DI, Rampuri R, Bown MJ, et al. Interleukin-6 receptor pathways in abdominal aortic aneurysm. Eur Heart J. 2013;34(48):3707-16

17. Fanola CL, Morrow DA, Cannon CP, Jarolim P, Lukas MA, Bode C, et al. I. Interleukin- 6 and the risk of adverse outcomes in patients after an acute coronary syndrome: observations from the SOLID-TIMI 52 (Stabilization of Plaque Using Darapladib-Thrombolysis in Myocardial Infarction 52) trial. J Am Heart Assoc. 2017;6(10):e005637.

18. Ridker PM, Libby P, MacFadyen JG, Thuren T, Ballantyne C, Fonseca F, et al. Modulation of the interleukin- 6 signalling pathway and incidence rates of atherosclerotic events and all-cause mortality: analyses from the Canakinumab Anti-Inflammatory Thrombosis Outcomes Study (CANTOS). Eur Heart J. 2018;39(38):3499-507.

19. Wei P, Han B, Zhang WJ, Bai J, Jiang CY, Qiu CR, et al. Effect of ticagrelor on the serum level of hs-CRP, ESM-1 and short-term prognosis of patients with acute STEMI. Exp Ther Med. 2017;13(2):604-8.

20. Gao CZ, Ma QQ, Wu J, Liu R, Wang F, Bai J, et al. Comparison of the Effects of ticagrelor and clopidogrel on inflammatory factors, vascular endothelium functions and short-term prognosis in patients with acute ST-Segment Elevation Myocardial Infarction Undergoing Emergency Percutaneous Coronary Intervention: a pilot study. Cell Physiol Biochem. 2018;48(1):385-96.

21. Husted S, Storey RF, Harrington RA, Emanuelsson H, Cannon CP. Changes in inflammatory biomarkers in patients treated with ticagrelor or clopidogrel. Clin Cardiol. 2010;33(4):206-12.

22. Ridker PM, Danielson E, Fonseca FA, Genest J, Gotto Jr AM, Kastelein JJP, et al. Reduction in C-reactive protein and LDL cholesterol and cardiovascular event rates after initiation of rosuvastatin: a prospective study of the JUPITER trial. Lancet. 2009;373(9670):1175-82.

23. Bohula EA, Giugliano RP, Cannon CP, Zhou J, Murphy SA, White JA, et al. Achievement of dual low-density lipoprotein cholesterol and high-sensitivity C-reactive protein targets more frequent with the addition of ezetimibe to simvastatin and associated with better outcomes in IMPROVE-IT. Circulation. 2015;132(13):1224-33.

24. Ridker PM, Lüscher TF. Anti-inflammatory therapies for cardiovascular disease. Eur Heart J. 2014;35(27):1782-91.

25. Ridker PM, Everett BM, Thuren T, MacFadyen JG, Chang WH, Ballantyne $C$, et al. Antiinflammatory therapy with canakinumab for atherosclerotic disease. N Engl J Med. 2017;377(12):1119-31.
26. Yagi R, Suzuki W, Seki N, Kohyama M, Inoue T, Arai T, et al. The IL-4 production capability of different strains of naive CD4(+) T cells controls the direction of the T(h) cell response. Int Immunol. 2002;14(1):1-11.

27. Peng H, Sarwar Z, Yang XP, Peterson EL, Xu J, Janic B, et al. Profibrotic Role for interleukin-4 in cardiac remodeling and dysfunction. Hypertension. 2015;66(3):582-9.

28. Gomez I, Duval V, Silvestre JS. Cardiomyocytes and macrophages discourse on the method to govern cardiac repair. Front Cardiovasc Med. 2018 Oct $2 ; 5: 134$.

29. Folco EJ, Sukhova GK, Quillard T, Libby P. Moderate hypoxia potentiates interleukin- $1 \beta$ production in activated human macrophages. Circ Res. $2014 ; 115(10): 875-83$.

30. Latz E, Xiao TS, Stutz A. Activation and regulation of the inflammasomes. Nat Rev Immunol. 2013;13(6):397-411.

31. Ahechu P, Zozaya G, Martí P, Hernández-Lizoáin JL, Baixauli J, Unamuno $X$, et al. NLRP3 inflammasome: a possible link between obesity-associated low-grade chronic inflammation and colorectal cancer development. Front Immunol. 2018 Dec 11;9:2918.

32. Vandanmagsar B, Youm YH, Ravussin A, Galgani JE, Stadler K, Mynatt RL, et al. The NLRP3 inflammasome instigates obesity-induced inflammation and insulin resistance. Nat Med. 2011;17(2):179-88.

33. Gabriel AS, Martinsson A, Wretlind B, Ahnve S. IL-6 levels in acute and post myocardial infarction: their relation to CRP levels, infarction size, left ventricular systolic function, and heart failure. Eur J Intern Med 2004;15(8):523-8.

34. Eskandari V, Amirzargar AA, Mahmoudi MJ, Rahnemoon Z, Rahmani F, Sadati S, et al. Gene expression and levels of IL-6 and TNFa in PBMCs correlate with severity and functional class in patients with chronic heart failure. Ir J Med Sci. 2018;187(2):359-68

35. Ridker PM. Clinician's guide to reducing inflammation to reduce atherothrombotic risk: JACC review topic of the week. J Am Coll Cardiol. 2018;72(25):3320-31.

36. Mahmoud AH, Taha NM, Zakhary M, Tadros MS. PTEN gene \& TNFalpha in acute myocardial infarction. Int J Cardiol Heart Vasc. $2019 \mathrm{Apr}$ $29 ; 23: 100366$

37. Orrem HL, Shetelig C, Ueland T, Limalanathan S, Nilsson PH, Husebye T, etal Soluble IL-1 receptor 2 is associated with left ventricular remodeling in patients with ST-elevation myocardial infarction. Int J Cardiol. 2018 Oct 1;268:187-92.

38. Lugrin J, Parapanov R, Rosenblatt-Velin N, Rignault-Clerc S, Feihl F, Waeber B et al. Interleukin-1 alpha is a crucial danger signal triggering acute myocardial inflammation during myocardial infarction. J Immunol. 2015;194(2):499-503.

39. Dutta P, Nahrendorf M. Regulation and consequences of monocytosis Immunol Rev. 2014;262(1):167-78.

40. Hanna A, Frangogiannis NG. The role of the TGF- $B$ superfamily in myocardial infarction. Front Cardiovasc Med. 2019 Sep 18;6:140. 